\title{
Structural basis for leucine-rich nuclear export signal recognition by CRM1
}

\author{
Xiuhua Dong ${ }^{1}$, Anindita Biswas ${ }^{1}$, Katherine E. Süel ${ }^{1}$, Laurie K. Jackson ${ }^{1}$, Rita Martinez ${ }^{1}$, \\ Hongmei Gu ${ }^{1}$, and Yuh Min Chook ${ }^{1}$ \\ ${ }^{1}$ Department of Pharmacology, University of Texas Southwestern Medical Center at Dallas, 6001 \\ Forest Park, Dallas, Texas 75390-9041, USA.
}

\section{Abstract}

CRM1 (also known as XPO1 and exportin 1) mediates nuclear export of hundreds of proteins through the recognition of the leucine-rich nuclear export signal (LR-NES). Here we present the 2.9 $\AA$ structure of CRM1 bound to snurportin 1 (SNUPN). Snurportin 1 binds CRM1 in a bipartite manner by means of an amino-terminal LR-NES and its nucleotide-binding domain. The LR-NES is a combined $a$-helical-extended structure that occupies a hydrophobic groove between two CRM1 outer helices. The LR-NES interface explains the consensus hydrophobic pattern, preference for intervening electronegative residues and inhibition by leptomycin $\mathrm{B}$. The second nuclear export signal epitope is a basic surface on the snurportin 1 nucleotide-binding domain, which binds an acidic patch on CRM1 adjacent to the LR-NES site. Multipartite recognition of individually weak nuclear export signal epitopes may be common to CRM1 substrates, enhancing CRM1 binding beyond the generally low affinity LR-NES. Similar energetic construction is also used in multipartite nuclear localization signals to provide broad substrate specificity and rapid evolution in nuclear transport.

Nuclear localization and export signals (NLSs and NESs) are recognized by karyopherin- $\beta$ (Kap- $\beta$, also known as importin and exportin) proteins to direct macromolecules in and out of the nucleus ${ }^{1-4}$. Although several classes of NLSs have been characterized ${ }^{5-8}$, only one class of NES, the LR-NES, is known at present ${ }^{9,10}$. The 10-15-residue LR-NESs were first identified in HIV-1 Rev and cyclic-AMP-dependent protein kinase inhibitor, and are recognized by the export-Kap- $\beta$ CRM1 (refs 9-16). Several mutagenesis and computational studies have led to a consensus sequence $\phi-X(2-3)-\phi-X(2-3)-\phi-X-\phi$ (in which $\phi$ is Lys, Val, Ile, Phe or Met, $\mathrm{X}$ is any amino acid, and the numbers in parentheses denote the number of repeats) that matches most known LR-NESs, but it is so broad that it is found in most helixcontaining proteins ${ }^{17-21}$. Because many proteins with this motif do not undergo nuclear export, other factors in the signal must affect its availability. Like the multipartite PY$\mathrm{NLS}^{8,22}$ and other vague organelle-targeting signals ${ }^{23,24}$, the LR-NES may be a complex and diverse signal that needs to be described not just by the consensus sequence but also in the structural and physical context of its interactions with CRM1.

(C2009 Macmillan Publishers Limited. All rights reserved

Correspondence and requests for materials should be addressed to Y.M.C. (yuhmin.chook@utsouthwestern.edu)..

Author Information Atomic coordinates and structure factors have been deposited in the Protein Data Bank under accession codes rcsb051649 and 3GB8.

Full Methods and any associated references are available in the online version of the paper at www.nature.com/nature.

Supplementary Information is linked to the online version of the paper at www.nature.com/nature. 
Antifungal antibiotic leptomycin B inhibits nuclear export by alkylating Cys 528 of human CRM1 (ref. 25). It is unclear if leptomycin B sterically blocks substrate binding or if it inhibits substrate binding by a conformational change. Effective inhibition of CRM1 by leptomycin B coupled with the availability of a LR-NES sequence motif have facilitated identification of hundreds of broadly functioning CRM1 substrates ${ }^{26,27}$. One such export substrate is snurportin 1 (SNUPN), which imports spliceosomal U small nuclear ribonucleoprotein particles (snRNPs) into the nucleus ${ }^{28}$. SNUPN contains an $N$-terminal importin- $\beta$ (karyopherin- $\beta$ )-binding domain (which is known as $\mathrm{SIBB}$ ) and a carboxy-terminal nucleotide-binding domain (NBD) ${ }^{29}$. The latter binds the $5^{\prime}-2,2,7$-terminal trimethylguanosine $\left(\mathrm{m}_{3} \mathrm{G}\right)$ cap of small nuclear RNAs (snRNAs) and the sIBB domain binds karyopherin- $\beta 1$ (Kap- $\beta 1$, also known as KPNB1 and importin- $\beta$ ) thus importing snRNP particles into the nucleus. CRM1 recycles nuclear SNUPN back to the cytoplasm ${ }^{28}$. Like other export Kap- $\beta$ s, CRM1 binds SNUPN and RanGTP cooperatively in the nucleus, and the CRM1-SNUPN-Ran complex is translocated through the nuclear pore complex. This ternary export complex probably forms through a stable CRM1-SNUPN binary intermediate $^{28}$. In the absence of atomic structures of CRM1-substrate complexes, the location of substrate-binding sites on CRM1, the physical nature of NES and its mechanism of recognition remain unknown.

Here we present the crystal structure of the CRM1-SNUPN complex. CRM1 is a ringshaped protein and SNUPN binds the central convex surface of CRM1 by its N-terminal LR-NES and NBD. The LR-NES is a combined a-helical-extended peptide that occupies a hydrophobic groove between the outer helices of CRM1 HEAT repeats 11 and 12. The LRNES interface explains the hydrophobic consensus and prevalence of intervening electronegative residues. The location of CRM1 Cys 528 in the hydrophobic groove indicates that leptomycin B inhibits substrate binding by the occupation of the LR-NESbinding site. The second NES epitope of SNUPN is the positively charged NBD surface that binds an acidic patch adjacent to the LR-NES groove. Multipartite recognition as observed in SNUPN may be used more generally to increase CRM1 binding beyond the usually low affinity LR-NES.

\section{Overall structure of the CRM1-SNUPN complex}

The 2.9 CRM1-SNUPN crystal structure was solved by multiwave-length anomalous dispersion (MAD) using native full-length human CRM1 (residues 1-1071) and residues 1342 of selenomethionine-labelled human SNUPN (Fig. 1a-c and Supplementary Table 1). The last 30 residues of SNUPN are dispensable for CRM1 binding (Supplementary Fig. 1) ${ }^{28}$ and SNUPN is exported in a CRM1-SNUPN-Ran complex that probably assembles through a stable CRM1-SNUPN intermediate (dissociation constant $\left(\mathrm{K}_{\mathrm{d}}\right) 1.4 \mu \mathrm{M}$; Supplementary Fig. 2) ${ }^{28}$. The final CRM1-SNUPN model has an $R_{\text {free }}$ of 0.269 and an $R_{\text {factor }}$ of 0.239 (Supplementary Table 1).

CRM1 has 20 HEAT repeats (H1-H20; Fig. 1a, b). A HEAT repeat generally contains two antiparallel helices A and B, each lining the convex and concave side of the protein. Most of repeat $\mathrm{H} 1$, several loops within $\mathrm{H} 1-\mathrm{H} 3, \mathrm{H} 8-\mathrm{H} 9$ and the last ten residues of CRM1 are not modelled owing to weak electron density. Repeats $\mathrm{H} 1-\mathrm{H} 20$ are arranged in a ring with $\mathrm{H} 2-$ H5 located within $10 \AA$ of $\mathrm{H} 20$. The outer and inner diameters of the CRM1 ring are approximately $90 \AA$ and $45 \AA$, respectively. H1-H19 are typical two-helical HEAT repeats but $\mathrm{H} 20$ has three helices, H20', H20A and H20B. Helix H20B is followed by a 27-residue helix named the $\mathrm{C}$-terminal or $\mathrm{C}$-helix that protrudes across the plane of the ring such that its C terminus contacts B helices of H8-H12 (Fig. 1a, b). Repeats H15-H20 adopt a similar conformation as in a C-terminal fragment of CRM1 (Protein Data Bank (PDB) accession 
1W9C; Ca root mean squared deviation (r.m.s.d.) 1.57 ), but the C-helix was not observed in that structure ${ }^{30}$.

Our SNUPN model includes the sIBB domain (residues 1-66) and NBD (residues 94-293). The CRM1-bound, nucleotide-free NBD is virtually identical to the isolated $\mathrm{m}_{3} \mathrm{G}$-cap-bound domain (PDB accession 1XK5; Ca r.m.s.d. 0.62 $)^{31}$. In contrast, CRM1-bound sIBB is quite different from Kap- $\beta 1$-bound $\operatorname{sIBB}^{32,33}$. Residues $1-10$ of the sIBB are helical and connected by a long loop to $\alpha$-helices $a 2^{\mathrm{IBB}}$ (residues 41-52) and $a 3^{\mathrm{IBB}}$ (residues 60-66) (Fig. 1c). Residues 1-17 protrude away from the protein to bind CRM1, whereas the rest of the sIBB drapes onto one face of the NBD (sIBB-NBD interaction buries $2,909 \AA^{2}$ surface area).

SNUPN binds in a bipartite manner to the outer surface of CRM1 that spans H11-H14. Residues 1-16, which form NES epitope I of SNUPN, interact with H11-H12 of CRM1 (Fig. 1a). The second binding element or NES epitope II, mostly loops surrounding the $\mathrm{m}_{3} \mathrm{G}$-cap-binding site, binds outer helices of CRM1 H12-H14. There are no direct contacts between the two NES epitopes, and their interactions with CRM1 bury an area of 3,551 $\AA^{2}$. Binding of SNUPN to the outer surface of CRM1 is notable as known Kap- $\beta$-substrate interactions, including that of export Kap- $\beta$ CSE1 (also known as CSE1L) (structural comparison in ref. 33), involve inner surfaces of the karyopherins ${ }^{34}$. Although Ran increases the CRM1-SNUPN affinity, mutagenesis data described later and structural comparison with the CSE1-Kap60-Ran (Kap60 is also known as Srp1 and KPNA1) export complex suggest that the binary and ternary CRM1 structures are similar ${ }^{33,35}$.

\section{NES epitope I of snurportin 1 is a classical LR-NES}

It was previously reported that SNUPN does not have a LR-NES, and binds CRM1 through residues 1-300 (sIBB and NBD) ${ }^{28}$. Structural examination of the CRM1-SNUPN intermediate and sequence analysis of SNUPN show that residues 1-16 (NES epitope I) contain a previously undetected LR-NES. Residues 4-14 of segment 1MEELSQALASSFSVSQ-16 match the $\phi-X(1-3)-\phi-X(2-3)-\phi-X-\phi$ LR-NES consensus.

Residues 1-10 of SNUPN form an amphipathic a-helix, residues 11-14 adopt an extended conformation and beyond that, residues 15-40 form a long loop (Figs 1c, 2a and Supplementary Fig. 3). The LR-NES (residues 1-16) is a structurally independent module that does not contact the NBD and protrudes away from the rest of SNUPN. Its N-terminal location that immediately precedes a long loop makes it highly accessible (Fig. 1c). Although no previous structure of this SNUPN segment is available, secondary structure prediction programs suggest that residues 2-9 are helical ${ }^{36,37}$. Discovery of the LR-NES in SNUPN is further supported by complementary biochemical and cellular localization studies (see later).

\section{Recognition of the leucine-rich NES by CRM1}

The LR-NES of SNUPN binds a hydrophobic groove between CRM1 helices H11A and H12A (buried surface area 1,770 $\AA^{2}$; Fig. 2 and Supplementary Fig. 3). Helices H11A and H12A are spaced farther apart (average distance between equivalent Cas is $14.5 \AA$ ) than other neighbouring A/B helix pairs in CRM1 (average distance between equivalent Cas is $11.5 \AA$ ), resulting in a prominent groove. This groove is wide at the $\mathrm{N}$-terminal end of the A helices, gradually narrows to a bottleneck at the third helical turn of the LR-NES and continues as a narrow groove to its $\mathrm{C}$-terminal end, which is capped by electrostatic interactions between Lys 534 and Glu 575 (Fig. 2a-c). The highly conserved groove is lined with pockets formed by hydro-phobic side chains from helices H11A, H11B and H12A (Fig. 
2b, c and Supplementary Fig. 4). Comparison with structures of unliganded CRM1 and other CRM1-LR-NES complexes will inform on structural flexibility of the groove.

SNUPN side chains Met 1, Leu 4, Leu 8, Phe 12 and Val 14 line one side of the LR-NES and are buried in the LR-NES binding groove by hydrophobic contacts with CRM1 (Fig. 2c and Supplementary Figs 3 and 5). The solvent accessible face of the LR-NES helix is composed of polar residues Glu 2, Glu 3, Ser 5, Gln 6 and Ser 10. Acidic NES side chains Glu 2 and Glu 3 make electrostatic contacts with basic CRM1 side chains Lys 560 and Lys 522 that flank the hydrophobic groove (Fig. 2a, c). Groove-flanking charged side chains on H11A and H12A also contact sIBB residues Ala 22, Tyr 31, Lys 34 and Tyr 25.

We examined interactions between the SNUPN LR-NES and CRM1 using in vitro pulldown assays, and compared them to interactions of previously identified LR-NESs. Deletion of the LR-NES helix in glutathione S-transferase (GST)-SNUPND(1-12) or double mutations of Leu 4 and Leu 8 to alanines abolished CRM1-SNUPN interactions, confirming the importance of these hydrophobic NES residues for CRM1 recognition (Fig. 3a). Mutation of electronegative residues Glu 2, Glu 3 and Ser 11 in the NES helix also decreased CRM1 interaction, suggesting a role for polar contacts at this interface.

The isolated SNUPN LR-NES bound CRM1 in the presence of RanGTP, demonstrating sufficiency of the LR-NES in CRM1 binding. The extent and pattern of SNUPN LR-NES binding was comparable to those of LR-NESs from HIV-1 Rev and NMD3 (sequences shown in Supplementary Fig. 6), indicative of their relatively low affinity for CRM1 (Fig. $3 b)^{10,38,39}$. Complementary LR-NES-binding site mutations of CRM1 quadruple mutant I521A/L525A/F561A/ F572A abolished binding to all three LR-NESs, confirming location of the LR-NES-binding site and importance of hydrophobic side chains in the groove. This mutant also confirmed that LR-NESs bind the same hydrophobic groove in the presence and absence of RanGTP. Thus, the binary CRM1-SNUPN complex informs on interactions in the ternary CRM1-SNUPN-Ran complex.

The LR-NES of SNUPN is also a functional export signal in cells. In nuclear transport competition assays in Saccharomyces cerevisiae ${ }^{12}$, reporter protein SV40 NLS-GFP-GFPSNUPN(1-15) was cytoplasmic even though SV40 NLS-GFP-GFP was nuclear. Mutation of SNUPN Leu 4 and Leu 8 to alanines abolished nuclear export in both the isolated LRNES and SNUPN (Fig. 4a). Thus, SNUPN(1-15) is a sufficient NES and Leu 4 and Leu 8 are necessary for efficient nuclear export of SNUPN. Furthermore, expression of NLS-GFPGFP-SNUPN(1-15) in xpo1-1 (or yeast CRM1) temperature-sensitive cells ${ }^{12}$ mislocalized the reporter to the nucleus at non-permissive temperature (Fig. 4b), confirming that nuclear export of the SNUPN LR-NES was mediated by CRM1.

\section{Structural and sequence features of a leucine-rich NES}

Available structures and secondary structure prediction of LR-NESs suggest that most LRNESs contain helices ${ }^{19}$. A notable structural feature of the SNUPN LR-NES is its helix to extended structural transition, which reflects the hydrophobic pattern of the consensus (Fig. $2 a)$. We labelled hydrophobic positions in the SNUPN LR-NES as $\phi 0, \phi 1, \phi 2, \phi 3$ and $\phi 4$ (consensus $\phi 1-X(2-3)-\phi 2-X(2-3)-\phi 3-X-\phi 4$, in which $\phi 0$ is Met 1 ; Fig. $2 b$ ). Hydrophobic pockets in the CRM1 groove that bind these LR-NES positions are similarly labelled. Positions $\phi 0-2$ (Met 1, Leu 4 and Leu 8) are on the same side of three consecutive a-helical turns, whereas $\phi 3$ and $\phi 4$ (Phe 12 and Val 14) have the alternating pattern characteristic of a $\beta$ strand (Fig. 2a-c).

Several structural features of the CRM1 hydrophobic groove seem to select for the combined helix-extended LR-NES conformation. First, the hydrophobic groove is wide at 
one end, allowing it to accommodate the LR-NES helix (Fig. 2a, b). Hydrophobic pockets here are appropriately spaced (5.4-6.0 $\AA$ ) to interact with hydrophobic residues on one side of an a-helix (Fig. 2b). Second, at the third LRNES helical turn, long CRM1 side chains Glu 529 and Lys 568 constrict the groove, necessitating a structural transition of the LR-NES to an extended conformation (Fig. 2b, c). Unsatisfied helical carbonyls at the break, backbone carbonyl and amide of the extended segment make polar contacts with CRM1 side chains Glu 529, Lys 537 and Lys 568 (Supplementary Fig. 7). Third, CRM1 pockets at the narrow part of the groove are spaced $\sim 8 \AA$ apart, matching the distance between side chains on one side of a $\beta$ strand.

The CRM1 groove contains three other pockets $\left(\phi 1^{\prime}, \phi 2^{\prime}\right.$ and $\left.\phi 4^{\prime}\right)$ adjacent to the $\phi 0, \phi 1, \phi 2$ and $\phi 4$ pockets (Fig. $2 b$ ). These pockets may provide further binding sites to accommodate the variety of hydro-phobic side chains and hydrophobic register observed in experimentally identified LR-NESs ${ }^{19,40}$. Location of the $\phi 1^{\prime}$ pocket between the $\phi 0$ and $\phi 1$ pockets, and the width of the groove here may also accommodate different LR-NES backbone conformations such as bent and $3_{10}$ helices, as well as the rare extended conformations, explaining the degeneracy observed at the $\mathrm{N}$-terminal of the consensus ${ }^{19}$.

A definite preference for glutamate, aspartate and serine residues has been observed in LRNES positions not occupied by hydrophobic residues ${ }^{19,40}$. One side of the LR-NES is exposed to solvent, explaining the presence of polar residues between the hydrophobic positions, whereas the basic surface that flanks the N-terminal half of the CRM1 groove explains the preference for acidic and electronegative residues (Fig. 2a). Previous sequence and structural analyses of LR-NES-containing proteins suggested that the signal needs to be accessible and flexible ${ }^{19}$. The N-terminal location of the LR-NES and its connection by a long loop to the rest of SNUPN makes it highly accessible. It will be critical to consider accessibility and structural context of LR-NESs within whole proteins in future efforts to identify the signal. LR-NESs at the termini of proteins and those flanked by long loops are more likely to be true export signals that can be exported by CRM1.

\section{The leptomycin B modification site}

Leptomycin B specifically inhibits CRM1 nuclear export ${ }^{13-15,27}$. The electrophilic a, $\beta$ unsaturated $\delta$-lactone of leptomycin B covalently binds the nucleophilic sulphydryl group of Cys 528 in CRM1 by a Michael-type addition ${ }^{25}$. Cys 528 is located in the LR-NES binding groove (Fig. 2c) and makes van der Waals contact with SNUPN Phe 12 (Supplementary Fig. 5). Therefore, covalent addition of leptomycin B to the sulphydryl of Cys 528 will block access to the LR-NES-binding site as part or the entire drug occupies the groove. Leptomycin B prevents formation of the ternary CRM1-SNUPN-Ran complex ${ }^{41}$. We show in Supplementary Fig. 8 that leptomycin B also prevents binary CRM1-SNUPN interaction in the absence of Ran, suggesting that the mode of LR-NES binding and inhibition is similar in the presence and absence of Ran.

\section{The C-terminal NES epitope II of SNUPN}

The interface between the NBD of SNUPN and H12-H14 of CRM1 is adjacent to the LRNES binding groove and constitutes the second NES site of the bipartite complex (buried surface area $1,490 \AA^{2}$; Fig. 5a-c). Many NBD residues at this interface are located near the empty $m_{3}$ G-cap-binding site. These include $126-V G K-128$ of the $\beta 2-\beta 3$ loop, 143 -

TKSGYCVN-150 of the $\beta 4-\beta 5$ loop, and Arg 278 of the $\beta 10-\alpha 4$ loop, which surround the nucleotide-binding site, as well as residues 178-EVNQ-181 and 221-KTKLNPF-227 on nearby $\beta 6-\beta 7$ and $a 2-\beta 8$ loops, respectively (Fig. 5b). NES epitope II of SNUPN interacts with CRM1 helices H13A, H14A, H14B and intra-HEAT loops of H12 and H13. Mutations 
of interface residues on either protein decreased CRM1-SNUPN interactions (Supplementary Fig. 9).

Interactions at the second NES site are mostly polar with a basic SNUPN surface complementing a CRM1 acidic interface (Fig. 5c). Electrostatic interactions here include a salt bridge between SNUPN Lys 144 and CRM1 Asp 624, and long-range electrostatic contacts between Lys 128, Arg 129, Lys 221 and Lys 223 of SNUPN, and Asp 624, Glu 683 and Asp 681 of CRM1. The acidic CRM1 interface is only a fraction of a large acidic patch that spans the entire convex surface of repeats H13-H20 (Supplementary Fig. 10). Given the bipartite nature of the CRM1-SNUPN interaction and proximity of a large acidic surface to the LR-NES binding site, we speculate that many CRM1 substrates contain basic NES epitopes in addition to LR-NES. These other NES epitopes may be conformational epitopes with a basic surface like the SNUPN NBD, or may be simpler linear epitopes that are enriched with basic residues.

The HIV-1 Rev protein may be another example of a multipartite CRM1 substrate. Fulllength Rev binds CRM1 100-fold more tightly than its LR-NES because of extra interactions with an adjacent basic element that contacts outer helices of H15 and H17 (refs 28,38). These results indicate that at least one additional NES epitope of Rev interacts with the Cterminal acidic surface located next to the LR-NES binding groove of CRM1 (Supplementary Fig. 9). Multipartite recognition by CRM1 would increase substrate affinity beyond that of isolated LR-NESs, which generally bind weakly ${ }^{21}$. We have previously shown that different NLS epitopes in the multipartite PY-NLS can contribute differently to total binding energy in different substrates, and combinatorial mixing of energetically weak and strong epitopes provide a mechanism to amplify signal diversity ${ }^{42}$. Similarly, combination of the LR-NES with further binding epitope(s) will also allow energetic variability of individual epitopes to generate NESs that are more diverse, evolvable and tunable. Thus, similar principles of substrate recognition seem to be common for both directions of nuclear trafficking.

\section{METHODS SUMMARY}

GST-CRM1 (human, residues 1-1071) and GST-SNUPN (human, residues 1-342) were expressed separately in Escherichia coli BL21(DE3) and purified separately by affinity and gel filtration chromatography. The purified proteins were mixed and the CRM1-SNUPN complex was purified by gel filtration chromatography. Crystals of the CRM1-SNUPN complex were obtained by vapour diffusion using $8 \%$ PEG 8000, $0.2 \mathrm{M}$ magnesium chloride, $0.1 \mathrm{M}$ MES, $\mathrm{pH} 6.5$, in the reservoir solution. The $2.9 \AA$ native data $(\lambda=$ $0.97934 \AA$ ) and the MAD data from a native CRM1-selenomethionine SNUPN crystal were collected at beam-line 19ID, Argonne National Laboratory and MAD phases obtained using Solve/Resolve ${ }^{43,44}$. The final model has $R_{\text {free }} 26.9 \%, R_{\text {factor }} 23.9 \%$ and contains CRM1 residues 53-57, 62-91, 96-115, 121-179, 187-387, 402-429 and 447-1061, and SNUPN residues $1-66$ and 94-293.

For in vitro pull down assays, $15 \mu \mathrm{g}$ GST-SNUPN or GST-NESs proteins were immobilized on $\sim 90 \mu \mathrm{l}$ glutathione sepharose (GE Healthcare) and incubated with $\sim 2.6 \mu \mathrm{M}$ of CRM1 proteins in the presence or absence of excess RanGTP. After extensive washing, bound proteins were separated by SDS-PAGE and Coomassie stained. To determine cellular localization of SNUPN proteins, S. cerevisiae BY4741 cells expressing NLS-GFP-GFP and NLS-GFP-GFP-SNUPN pRS415 plasmids were analysed as previously described ${ }^{42}$. pRS416 NLS-GFP-GFP-SNUPN(1-15) was transformed into the xpo1-1 strain (plasmid KWY121; ref. 12) and BY4741 cells for the temperature-sensitive cell assay. 


\section{METHODS}

\section{CRM1-SNUPN complex}

GST-CRM1 (human, residues 1-1071) and GST- SNUPN (human, residues 1-342) were expressed separately in E. coli BL21(DE3) (Stratagene) at $25^{\circ} \mathrm{C}$ and purified separately. The cells were lysed in buffer A (50 mM Tris, pH 7.5, $200 \mathrm{mM}$ sodium chloride, $1 \mathrm{mM}$ EDTA, $2 \mathrm{mM}$ DTT, 20\% glycerol) followed by affinity purification with glutathione sepharose (GE Healthcare), cleavage off the column with TEV protease and gel filtration chromatography. CRM1-SNUPN mixed at 2:3 molar ratio was purified by gel filtration chromatography. Selenomethionine SNUPN was expressed in BL21(DE3) cells using M9 minimal media, purified as for native SNUPN and the native CRM1-selenomethionine SNUPN complex was purified as for the native complex.

\section{X-ray crystallography}

Crystals of the CRM1-SNUPN complex ( $P 6_{4} 22, a=b=250.4 \AA$, $c=190.1 \AA$, one complex per asymmetric unit; $76 \%$ solvent) were obtained by vapour diffusion in hanging drops using 8\% PEG 8000, 0.2 M magnesium chloride, 0.1M MES, pH 6.5, in the reservoir solution at $18{ }^{\circ} \mathrm{C}$ and flash-frozen in liquid propane. Selenomethionine crystals were of the same space group and similar unit cell parameters. The $2.9 \AA$ native data $(\lambda=0.97934 \AA)$ and the MAD data $\left(\lambda_{\text {peak }}=0.97935 \AA, \lambda_{\text {inflection }}=0.97945 \AA, \lambda_{\text {remote }}=0.97167 \AA\right)$ were collected at $100 \mathrm{~K}$ at beamline 19ID, Argonne National Laboratory and processed with HKL2000 (ref. 45). Seven out of ten possible selenium sites were found using the programs Shel ${ }^{46}$ and Solve ${ }^{44}$. Heavy atom refinement and MAD phasing were carried out using Solve/Resolve ${ }^{43,44}$. Iterative cycles of model building using program $\mathrm{COOT}^{47}$ and refinement using $\mathrm{CNS}^{48}$ and a final TLS refinement ${ }^{49}$, all using the same test data set, produced a final model ( $R_{\text {free }} 26.9 \%, R_{\text {work }} 23.9 \%$ ) that contains CRM1 residues 53-57, 6291, 96-115, 121-179, 187-387, 402-429 and 447-1061 and SNUPN residues 1-66 and 94293. Average B-factor values for the model are $100.3 \AA^{2}$ for repeats H1 and H2 of CRM1, $37.2 \AA^{2}$ for H3-H20A of CRM1, $104.1 \AA^{2}$ for H20B to the C terminus of CRM1 and $26.8 \AA^{2}$ for SNUPN. Wilson B-factors of the data $\left(64.6 \AA^{2}\right.$ for the MAD inflection data) are comparable to average B-factor of the model $\left(39.3 \AA^{2}\right)$.

\section{In vitro binding assays}

Site-directed mutagenesis of SNUPN and CRM1 were performed using the QuickChange method (Stratagene) and all constructs were sequenced. The SNUPN(1-15) NES as well as NESs of Rev and Nmd3 were generated by ligation of annealed oligonucleotides into the pGEX-Tev vector. Approximately $15 \mu \mathrm{g}$ of immobilized GST-SNUPN or GST-NESs proteins (glutathione sepharose; GE Healthcare) were incubated with $\sim 2.6 \mu \mathrm{M}$ of CRM1 proteins in the presence or absence of RanGTP (fivefold molar excess). After extensive washing, bound proteins were visualized by SDS-PAGE and Coomassie staining.

\section{Isothermal titration calorimetry experiments}

The CRM1-SNUPN experiment was performed twice, with 18 and 22 injections of $5 \mu 1$ SNUPN(1-342) (290-386 $\mu \mathrm{M})$ into $1.4 \mu \mathrm{l}$ of CRM1 (12-14 $\mu \mathrm{M})$ at $15^{\circ} \mathrm{C}$. The CRM1Ran experiment was performed once with 22 injections of $5 \mu 1$ RanGppNHp $(290 \mu \mathrm{M})$ into $1.4 \mathrm{ml}$ of CRM1 $(12 \mu \mathrm{M})$ at $15^{\circ} \mathrm{C}$. All proteins were in buffer containing $50 \mathrm{mM}$ Tris, $\mathrm{pH}$ $7.5,145 \mathrm{mM}$ sodium chloride, $5 \mathrm{mM}$ magnesium chloride and $8 \%$ glycerol. The heat of dilution obtained from injecting a ligand into buffer was subtracted before $K_{\mathrm{d}}$ and binding ratio were calculated using the ORIGIN data analysis software (Microcal, Inc.). The chisquare value for each CRM1-SNUPN experiment is relatively low $\left(7.907 \times 10^{4}\right.$ and $1.410 \times$ $10^{5}$, respectively) indicating the quality of the fit. In addition, the error (s.d.) reported in 
Supplementary Fig. 2 demonstrates the reproducibility of the isothermal titration calorimetry (ITC) experiments. ITC experiments were performed at $15{ }^{\circ} \mathrm{C}$ because CRM1 was more stable at a lower temperature. The CRM1-SNUPN experiment was repeated at $23{ }^{\circ} \mathrm{C}$ the data obtained at the two temperatures are similar $\left(K_{\mathrm{d}}=6.5 \mu \mathrm{M}, \Delta \mathrm{H}=-19.1 \mathrm{kcal} \mathrm{mol}^{-1}\right.$ and $\left.\mathrm{T} \Delta \mathrm{S}=-12.1 \mathrm{kcal} \mathrm{mol}^{-1}\right)$. The fourfold weaker binding affinity at $23{ }^{\circ} \mathrm{C}$ probably reflects decreased stability of CRM1 at higher temperature.

\section{In vivo localization studies}

To clone the NLS-GFP-GFP and NLS-GFP-GFP-SNUPN plasmids, two GFP genes were cloned into a modified pRS415 (CEN6, ARS, LEU2 and $A P^{R}$ ) shuttle vector containing a $5^{\prime}$ ADH1 promoter ${ }^{50}$. The SV40 NLS (PKKKRKV) was inserted upstream of the GFP genes using the Quikchange (Stratagene) method. SNUPN gene was cloned 3' of the GFP genes and GGSGG linkers were inserted by Quikchange between all four genes. The whole NLSGFP-GFP-SNUPN(1-15) insert was also subcloned into a pRS416 (CEN6, ARS, $U R A 3$ and $A P^{R}$ ) shuttle vector.

For the transport competition assay, BY4741 cells expressing pRS415 plasmids were grown overnight at $30^{\circ} \mathrm{C}$ in SC-Leu media and analysed as previously reported ${ }^{42}$. For the temperature-sensitive cell assay, pRS416 NLS-GFP-GFP-SNUPN(1-15) was transformed into the xpo1-1 strain (plasmid KWY121; ref. 12) and BY4741 cells. Cells were grown overnight at room temperature and analysed as above. After the fluorescence was examined at room temperature, the temperature in the incubation chamber was increased to $37{ }^{\circ} \mathrm{C}$ for an hour and reporter fluorescence was examined in the same cells.

\section{Supplementary Material}

Refer to Web version on PubMed Central for supplementary material.

\section{Acknowledgments}

We thank Y. Sheng, C. Kong, D. Tomchick and C. Dann for assistance during data collection and structure determination, S. Padrick for advice on fluorescence binding assays, G. Süel and T. Cagatay for help with microscopy, K. Weis for yeast strains and constructs, X. Zhang, L. Rice and M. Rosen for discussion. This work is funded by National Institute of Health (NIH) grants R01GM069909, R01GM069909-03S1, 5-T32-GM008297, Welch Foundation grant I-1532 and the UT Southwestern Endowed Scholars Program. Use of the Argonne National Laboratory Stuctural Biology Center beamlines at the Advanced Photon Source, was supported by the US Department of Energy, Office of Energy Research, under contract no. W-31-109-ENG-38.

\section{References}

1. Tran EJ, Bolger TA, Wente SR. SnapShot: nuclear transport. Cell. 2007; 131:420. [PubMed: 17956740]

2. Weis K. Regulating access to the genome: nucleocytoplasmic transport throughout the cell cycle. Cell. 2003; 112:441-451. [PubMed: 12600309]

3. Gorlich D, Kutay U. Transport between the cell nucleus and the cytoplasm. Annu. Rev. Cell Dev. Biol. 1999; 15:607-660. [PubMed: 10611974]

4. Conti E, Izaurralde E. Nucleocytoplasmic transport enters the atomic age. Curr. Opin. Cell Biol. 2001; 13:310-319. [PubMed: 11343901]

5. Dingwall C, Sharnick SV, Laskey RA. A polypeptide domain that specifies migration of nucleoplasmin into the nucleus. Cell. 1982; 30:449-458. [PubMed: 6814762]

6. Lanford RE, Butel JS. Construction and characterization of an SV40 mutant defective in nuclear transport of T antigen. Cell. 1984; 37:801-813. [PubMed: 6086146]

7. Kalderon D, Richardson WD, Markham AF, Smith AE. Sequence requirements for nuclear location of simian virus 40 large-T antigen. Nature. 1984; 311:33-38. [PubMed: 6088992] 
8. Lee BJ, et al. Rules for nuclear localization sequence recognition by karyopherin $\beta 2$. Cell. 2006; 126:543-558. [PubMed: 16901787]

9. Wen W, Meinkoth JL, Tsien RY, Taylor SS. Identification of a signal for rapid export of proteins from the nucleus. Cell. 1995; 82:463-473. [PubMed: 7634336]

10. Fischer U, et al. The HIV-1 Rev activation domain is a nuclear export signal that accesses an export pathway used by specific cellular RNAs. Cell. 1995; 82:475-483. [PubMed: 7543368]

11. Richards SA, Carey KL, Macara IG. Requirement of guanosine triphosphate-bound ran for signalmediated nuclear protein export. Science. 1997; 276:1842-1844. [PubMed: 9188526]

12. Stade K, Ford CS, Guthrie C, Weis K. Exportin 1 (Crm1p) is an essential nuclear export factor. Cell. 1997; 90:1041-1050. [PubMed: 9323132]

13. Fornerod M, Ohno M, Yoshida M, Mattaj IW. CRM1 is an export receptor for leucine-rich nuclear export signals. Cell. 1997; 90:1051-1060. [PubMed: 9323133]

14. Ossareh-Nazari B, Bachelerie F, Dargemont C. Evidence for a role of CRM1 in signal-mediated nuclear protein export. Science. 1997; 278:141-144. [PubMed: 9311922]

15. Fukuda M, et al. CRM1 is responsible for intracellular transport meditted by the nuclear export signal. Nature. 1997; 390:308-311. [PubMed: 9384386]

16. Neville M, et al. The importin- $\beta$ family member Crm1p bridges the interaction between Rev and the nuclear pore complex during nuclear export. Curr. Biol. 1997; 7:767-775. [PubMed: 9368759]

17. Bogerd HP, et al. Protein sequence requirements for function of the human T-cell leukemia virus type 1 Rex nuclear export signal delineated by a novel in vivo randomization-selection assay. Mol. Cell. Biol. 1996; 16:4207-4214. [PubMed: 8754820]

18. Henderson BR, Eleftheriou A. A comparison of the activity, sequence specificity, and CRM1dependence of different nuclear export signals. Exp. Cell Res. 2000; 256:213-224. [PubMed: 10739668]

19. la Cour T, et al. Analysis and prediction of leucine-rich nuclear export signals. Protein Eng. Des. Sel. 2004; 17:527-536. [PubMed: 15314210]

20. Kutay U, Guttinger S. Leucine-rich nuclear-export signals: born to be weak. Trends Cell Biol. 2005; 15:121-124. [PubMed: 15752974]

21. Engelsma D, Bernad R, Calafat J, Fornerod M. Supraphysiological nuclear export signals bind CRM1 independently of RanGTP and arrest at Nup358. EMBO J. 2004; 23:3643-3652. [PubMed: 15329671]

22. Cansizoglu AE, et al. Structure-based design of a pathway-specific nuclear import inhibitor. Nature Struct. Mol. Biol. 2007; 14:452-454. [PubMed: 17435768]

23. Mancias JD, Goldberg J. Exiting the endoplasmic reticulum. Traffic. 2005; 6:278-285. [PubMed: 15752134]

24. Swanton E, High S. ER targeting signals: more than meets the eye? Cell. 2006; 127:877-879. [PubMed: 17129773]

25. Kudo N, et al. Leptomycin B inactivates CRM1/exportin 1 by covalent modification at a cysteine residue in the central conserved region. Proc. Natl Acad. Sci. USA. 1999; 96:9112-9117. [PubMed: 10430904]

26. Matsuyama A, et al. ORFeome cloning and global analysis of protein localization in the fission yeast Schizosaccharomyces pombe. Nature Biotechnol. 2006; 24:841-847. [PubMed: 16823372]

27. Nishi K, et al. Leptomycin B targets a regulatory cascade of crm1, a fission yeast nuclear protein, involved in control of higher order chromosome structure and gene expression. J. Biol. Chem. 1994; 269:6320-6324. [PubMed: 8119981]

28. Paraskeva E, et al. CRM1-mediated recycling of snurportin 1 to the cytoplasm. J. Cell Biol. 1999; 145:255-264. [PubMed: 10209022]

29. Huber J, et al. Snurportin1, an m3G-cap-specific nuclear import receptor with a novel domain structure. EMBO J. 1998; 17:4114-4126. [PubMed: 9670026]

30. Petosa C, et al. Architecture of CRM1/Exportin1 suggests how cooperativity is achieved during formation of a nuclear export complex. Mol. Cell. 2004; 16:761-775. [PubMed: 15574331] 
31. Strasser A, Dickmanns A, Luhrmann R, Ficner R. Structural basis for m3G-cap-mediated nuclear import of spliceosomal UsnRNPs by snurportin1. EMBO J. 2005; 24:2235-2243. [PubMed: 15920472]

32. Mitrousis G, Olia AS, Walker-Kopp N, Cingolani G. Molecular basis for the recognition of snurportin 1 by importin beta. J. Biol. Chem. 2008; 283:7877-7884. [PubMed: 18187419]

33. Dong X, Biswas A, Chook YM. Structural basis of assembly and disassembly of the CRM1 nuclear export complex. Nature Struct. Mol. Biol. doi:10.1038/nsmb.1585 (in the press).

34. Suel KE, Cansizoglu AE, Chook YM. Atomic resolution structures in nuclear transport. Methods. 2006; 39:342-355. [PubMed: 16938467]

35. Matsuura Y, Stewart M. Structural basis for the assembly of a nuclear export complex. Nature. 2004; 432:872-877. [PubMed: 15602554]

36. Jones DT. Protein secondary structure prediction based on position-specific scoring matrices. J. Mol. Biol. 1999; 292:195-202. [PubMed: 10493868]

37. Meiler J, Baker D. Coupled prediction of protein secondary and tertiary structure. Proc. Natl Acad. Sci. USA. 2003; 100:12105-12110. [PubMed: 14528006]

38. Askjaer P, et al. The specificity of the CRM1-Rev nuclear export signal interaction is mediated by RanGTP. J. Biol. Chem. 1998; 273:33414-33422. [PubMed: 9837918]

39. Thomas F, Kutay U. Biogenesis and nuclear export of ribosomal subunits in higher eukaryotes depend on the CRM1 export pathway. J. Cell Sci. 2003; 116:2409-2419. [PubMed: 12724356]

40. Kosugi S, Hasebe M, Tomita M, Yanagawa H. Nuclear export signal consensus sequences defined using a localization-based yeast selection system. Traffic. 2008; 9:2053-2062. [PubMed: 18817528]

41. Englmeier L, et al. RanBP3 influences interactions between CRM1 and its nuclear protein export substrates. EMBO Rep. 2001; 2:926-932. [PubMed: 11571268]

42. Suel KE, Gu H, Chook YM. Modular organization and combinatorial energetics of proline-tyrosine nuclear localization signals. PLoS Biol. 2008; 6:e137. [PubMed: 18532879]

43. Terwilliger TC. Maximum-likelihood density modification. Acta Crystallogr. D. 2000; 56:965972. [PubMed: 10944333]

44. Terwilliger TC, Berendzen J. Automated MAD and MIR structure solution. Acta Crystallogr. D. 1999; 55:849-861.

45. Otwinowski Z, Minor W. Processing of X-ray diffraction data collected in oscillation mode. Methods Enzymol. 1997; 276:307-326.

46. Sheldrick GM. A short history of SHELX. Acta Crystallogr. A. 2008; 64:112-122. [PubMed: 18156677]

47. Emsley P, Cowtan K. Coot: model-building tools for molecular graphics. Acta Crystallogr. D. 2004; 60:2126-2132. [PubMed: 15572765]

48. Brunger AT, et al. Crystallography \& NMR system: A new software suite for macromolecular structure determination. Acta Crystallogr. D. 1998; 54:905-921. [PubMed: 9757107]

49. Winn MD, Isupov MN, Murshudov GN. Use of TLS parameters to model anisotropic displacements in macromolecular refinement. Acta Crystallogr. D. 2001; 57:122-133. [PubMed: 11134934]

50. Sikorski RS, Hieter P. A system of shuttle vectors and yeast host strains designed for efficient manipulation of DNA in Saccharomyces cerevisiae. Genetics. 1989; 122:19-27. [PubMed: 2659436] 
a
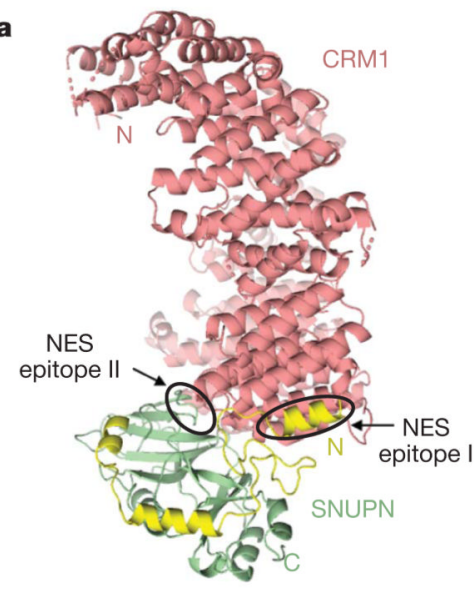

b

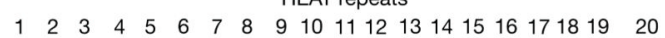

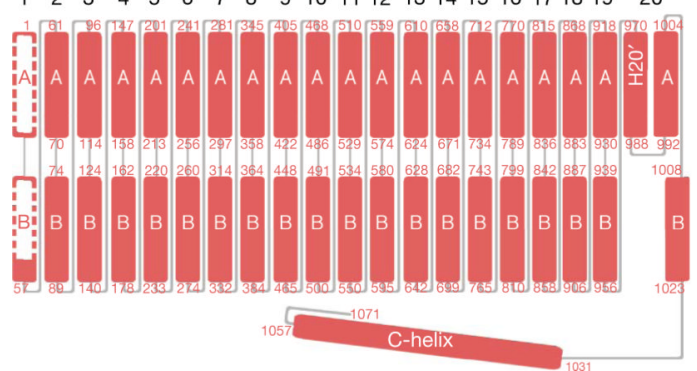

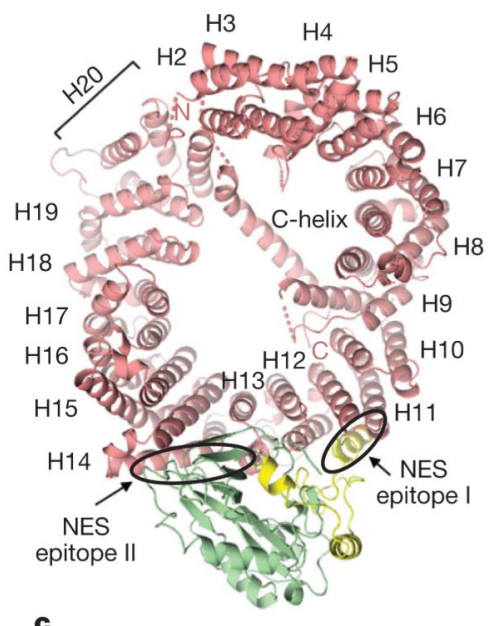

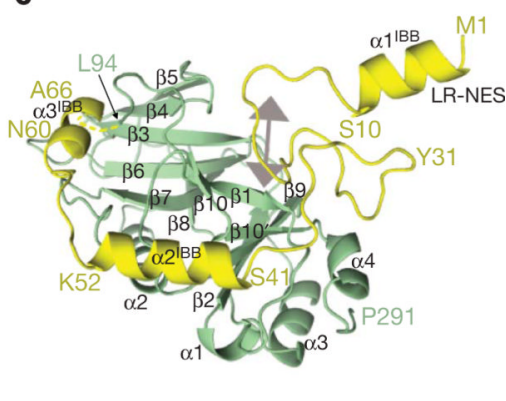

Figure 1. Overall structure of the CRM1-SNUPN complex

a, Orthogonal views of the CRM1-SNUPN complex. CRM1 is pink and the sIBB and NBD domains of SNUPN are yellow and green, respectively. HEAT repeats 2-20 of CRM1 are labelled H2-H20. b, HEAT repeat organization of CRM1. Most of H1 is disordered and not modelled in the structure. c, Structure of CRM1-bound SNUPN. The linker between sIBB and NBD of SNUPN is shown as a dashed yellow line and a grey arrow marks the location of the nucleotide-binding site of this nucleotide-free SNUPN. 

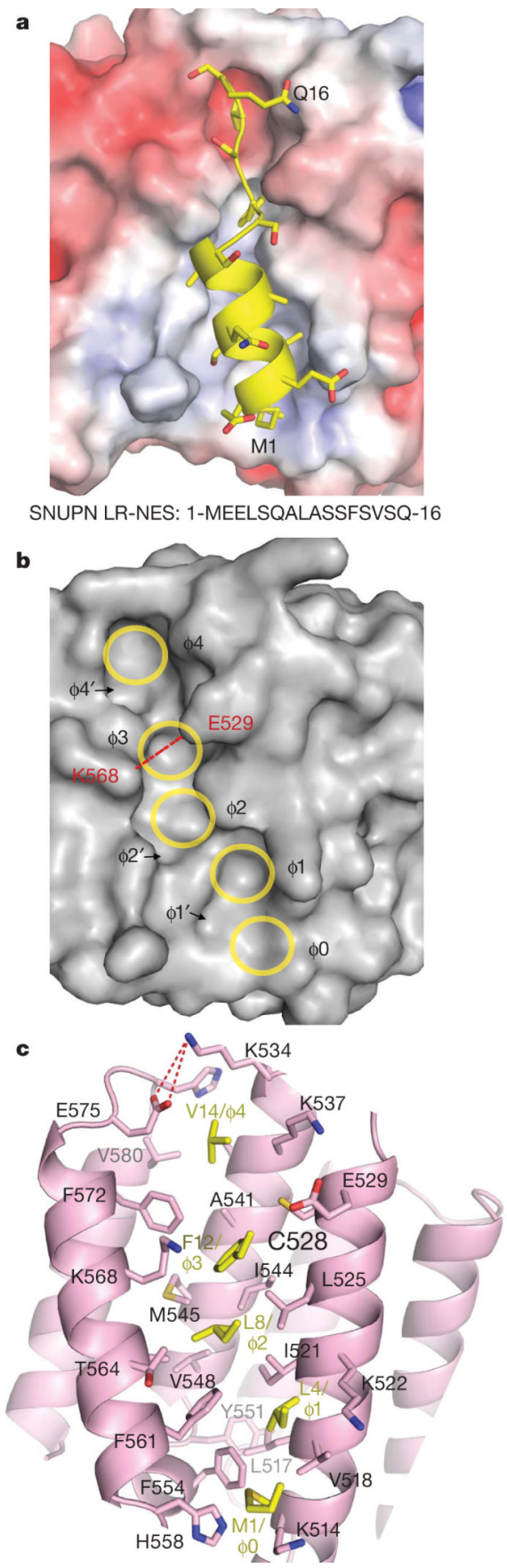

Figure 2. The LR-NES-binding site

a, The LR-NES interface showing the electrostatic surface potential of CRM1 and a ribbon diagram of the SNUPN LR-NES. The amino-acid sequence of the LR-NES of SNUPN is shown. b, Molecular surface of the LR-NES binding groove. Individual pockets that bind the five hydrophobic residues of the SNUPN LR-NES are outlined in yellow. Additional pockets are labelled and a dashed line is drawn between Lys 568 and Glu 529 at the narrowest point of the groove. c, Residues in the hydrophobic groove of CRM1 (pink) and hydrophobic residues of the SNUPN LR-NES (yellow). 

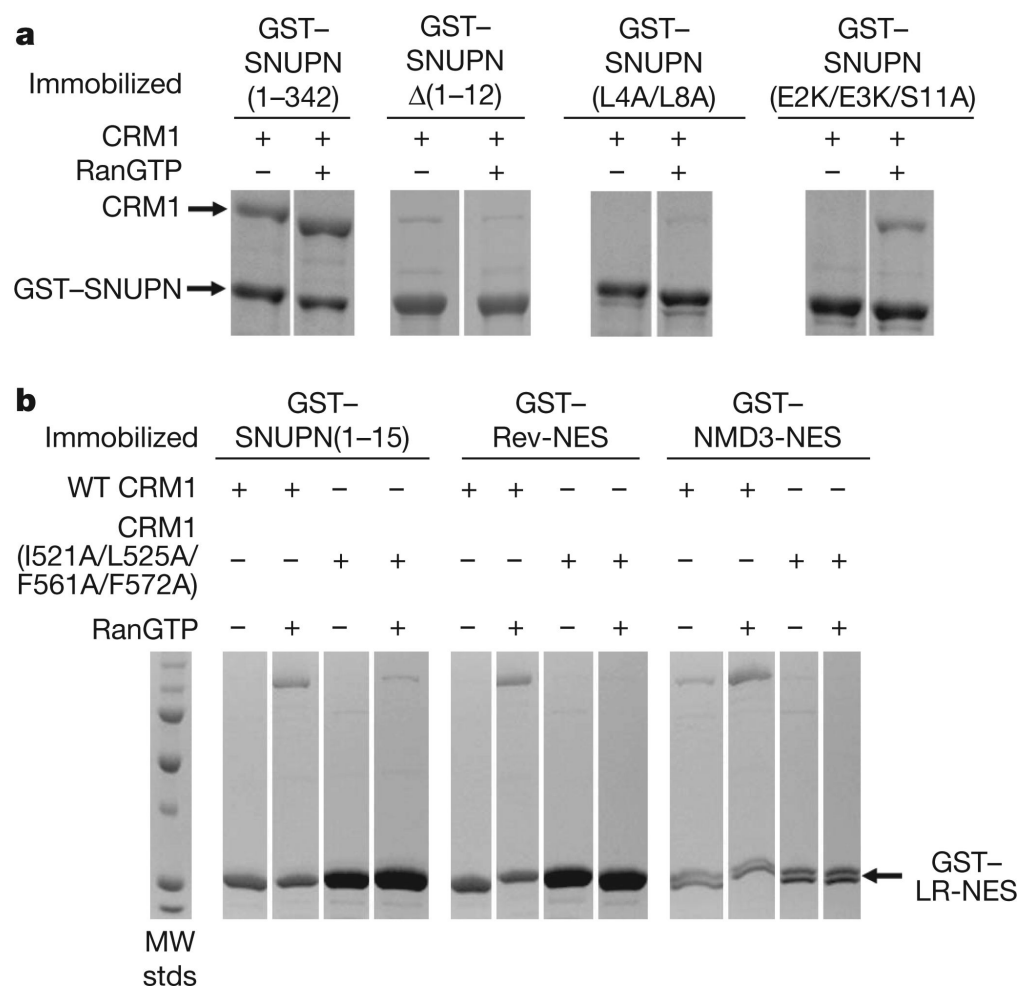

Figure 3. Hydrophobic residues are critical for LR-NES recognition

a, Mutations in the LR-NES of SNUPN that affected CRM1 binding. Deletion of the LRNES (SNUPN $\Delta(1-12)$ ) and mutation of residues Leu 4 and Leu 8 (SNUPN(L4A/L8A)) abolished interaction with CRM1. Mutation of electronegative residues of the LR-NES (SNUPN(E2K/E3K/S11A)) also decreased CRM1 binding. Bound proteins in the in vitro pull-down assays of immobilized GST-SNUPN proteins and CRM1 were visualized by SDS-PAGE and Coomassie staining. b, Mutations of hydrophobic residues on CRM1 helices H11A and H12A disrupt interaction with LR-NESs. Immobilized GST-NESs of SNUPN, HIV-1 Rev and NMD3 were incubated with either wild-type (WT) CRM1 or mutant CRM1(I521A/L525A/F561A/ F572A) in the presence and absence of RanGTP. Bound proteins were resolved with SDS-PAGE and Coomassie staining. MW stds, molecular weight standards. 
a

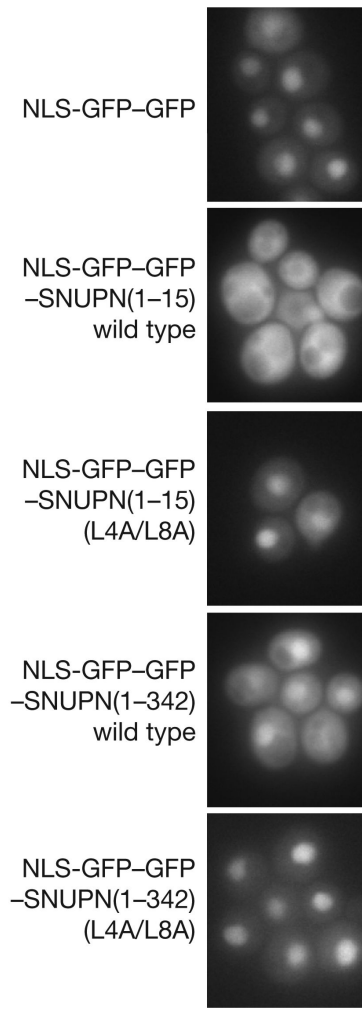

b

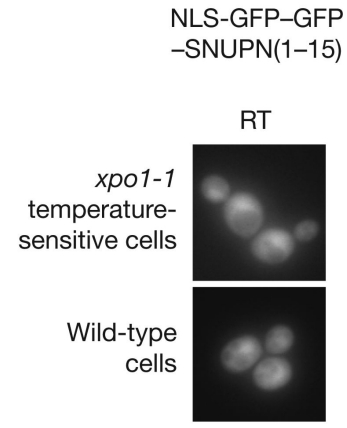

$37^{\circ} \mathrm{C}$

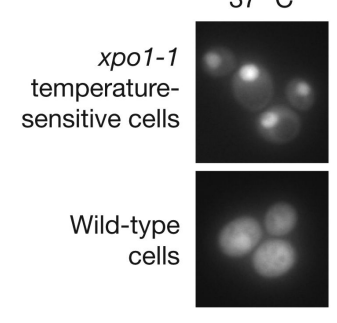

Figure 4. Localization of the SNUPN and its LR-NES in yeast

a, The NLSGFP-GFP-SNUPN reporter is localized to the cytoplasm in the wild-type $S$. cerevisiae cells, but the NLS-GFP-GFP-SNUPN(L4A/L8A) mutant accumulates in the nucleus. Each panel displays the same GFP fluorescence scale. b, Location of NLS-GFPGFP-SNUPN-NES in xpo1-1 temperature-sensitive (top) and wild-type (bottom) cells at the permissive (room temperature, RT) and non-permissive $\left(37^{\circ} \mathrm{C}\right)$ temperature. The reporter accumulates in the nucleus of xpo1-1 temperature-sensitive cells at the non-permissive temperature, but remains cytoplasmic in the wild-type cells. 
a

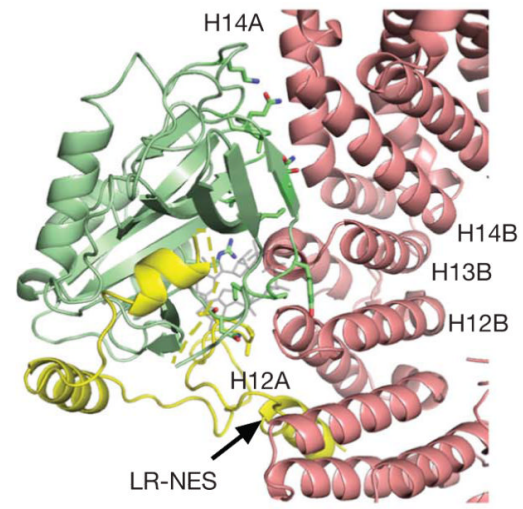

b
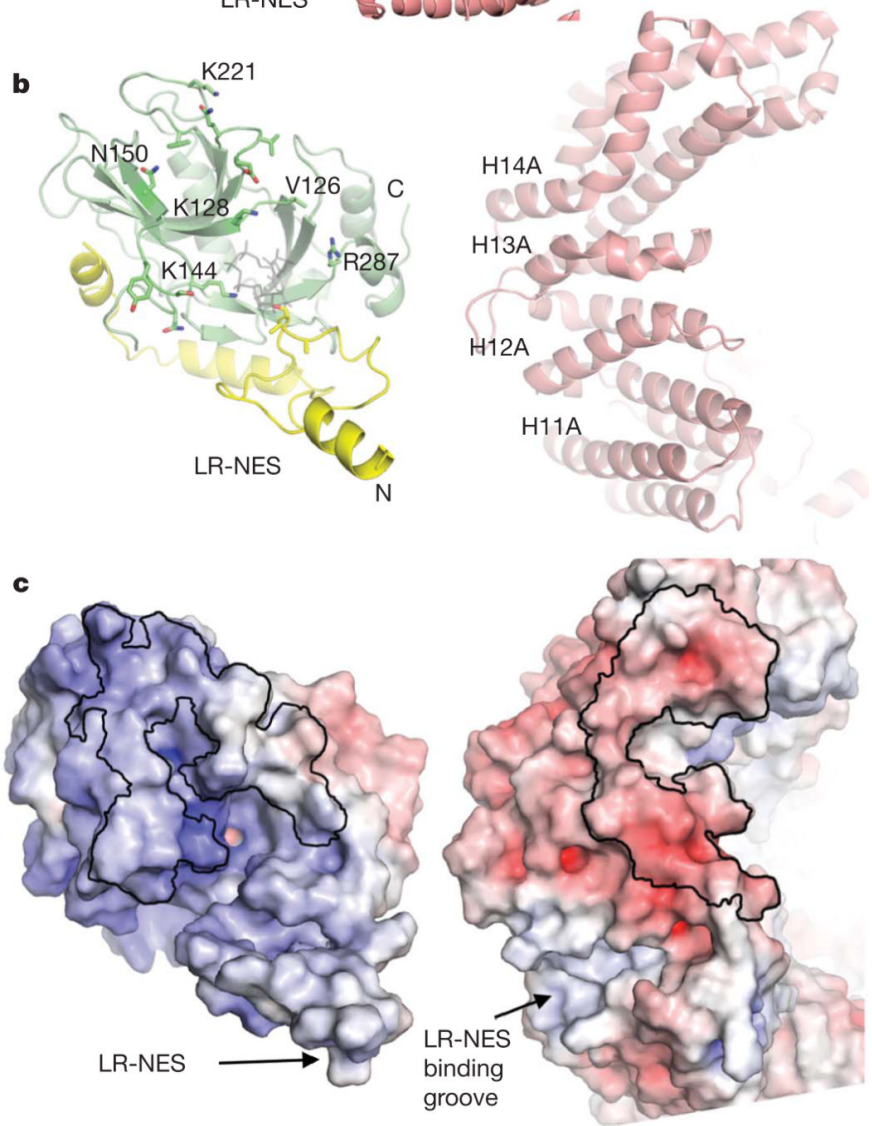

Figure 5. Interactions of CRM1 with the SNUPN NES epitope II

a, Ribbon drawing of the interface between CRM1 (pink) and the NES epitope II of SNUPN, which is found on its NBD (green). The N-terminal sIBB domain of SNUPN is in yellow. No nucleotide is bound in the CRM1-SNUPN complex but the $\mathrm{m}_{3} \mathrm{G}$-cap nucleotide from superimposed nucleotide-bound NBD (PDB accession 1XK5) is drawn in grey to show the location of the nucleotide-binding site. $\mathbf{b}, \mathbf{c}$, SNUPN and CRM1 in a are pried apart to show the interface (outlined with black lines) and electrostatic surface potential (scale of -12 $\mathrm{kT} \mathrm{e}^{-1}$ to $+12 \mathrm{kT} \mathrm{e}^{-1}$ ) of individual proteins at the interface. 